\title{
The increase of the ionospheric activity as measured by GPS
}

\author{
René Warnant and Eric Pottiaux \\ Royal Observatory of Belgium, Avenue Circulaire, 3, B-1180 Brussels, Belgium \\ (Received December 31, 1999; Revised September 25, 2000; Accepted September 25, 2000)
}

\begin{abstract}
The paper outlines a method allowing to compute the TEC with a precision of about 2-3 TECU and to detect Travelling Ionospheric Disturbances using GPS measurements. We describe the solar cycle dependance of the TEC and TIDs. Since the beginning of 1998, we have observed a stronger ionospheric activity due to the increasing solar activity. This ionospheric activity is characterized by larger TEC values which are regularly reaching the level of 60 TECU and by a larger number of Travelling Ionospheric Disturbances. During the winter 1999-2000, the mean daily TEC was above 45 TECU; at solar minimum the mean daily TEC is ranging from 4 TECU to 12 TECU. In January 2000 (close to solar maximum) more than 1300 events due to TID's were detected: it is 6.5 more than in January 1996 (at solar minimum).
\end{abstract}

\section{Introduction}

The ionospheric refraction remains a major error source in GPS positioning, in particular for real-time applications. This effect is usually reduced by forming single differences between one-way phase or code observations collected by a minimum of 2 GPS receivers. The residual ionospheric error remaining in the single differences depends on two parameters: the Total Electron Content (TEC) and its gradients in space (Wanninger, 1994; Warnant, 1996). The Total Electron Content is very variable in space and time: it is a function of geomagnetic latitude, local time, season, ... In particular, several studies demonstrated that the TEC strongly depends on solar activity (da Rosa et al., 1973; Soicher, 1988; Feitcher and Leitinger, 1997; Van Velthoven, 1990). The maximum of the 11-year solar activity cycle will be reached in 2000 or 2001. During this period of high solar activity, the ionosphere will have a stronger influence on GPS positioning. For this reason, we decided to develop a software to monitor the ionospheric activity in order to understand its impact on GPS. The idea of this work is to build a data set which can serve as reference to identify possible correlations between the ionospheric activity and unidentified problems in GPS time series or GPS data processing. Such correlations between the ionosphere and Transit position time series were reported by Dehant and Pâquet (1983).

The Global Positioning System has already proved to be a very useful tool to study the ionosphere. Indeed, GPS code and carrier phase measurements can be processed in order to determine the Total Electron Content (Lanyi and Roth, 1988; Warnant, 1996).

\section{Computing the TEC Using GPS Measurements} In practice, the TEC can be obtained from:

1) the so-called geometry-free combination of dual fre-

Copy right $(\mathrm{C}$ The Society of Geomagnetism and Earth, Planetary and Space Sciences (SGEPSS); The Seismological Society of Japan; The Volcanological Society of Japan; The Geodetic Society of Japan; The Japanese Society for Planetary Sciences. quency code measurements, $P_{p, G F}^{i}$;

$$
P_{p, G F}^{i}=P_{p, L 1}^{i}-P_{p, L 2}^{i}
$$

This equation can be rewritten in function of the Total Electron Content, $\mathrm{TEC}_{p}^{i}$ :

$$
P_{p, G F}^{i}=-0.105 \mathrm{TEC}_{p}^{i}+\left(D G_{p}-D G^{i}\right)
$$

with $\mathrm{TEC}_{p}^{i}$ slant TEC in TEC Units (or TECU) measured along the path going from satellite $i$ to receiver $p$; 1 TECU $=10^{16}$ electrons $\mathrm{m}^{-2}$;

$D G^{i}, D G_{p}$ the satellite $i$ and receiver $p$ differential group delays (in metres);

$P_{p, L 1}^{i}, P_{p, L 2}^{i}$ the $L 1, L 2 P$-code measurements (in metres) made by receiver $p$ on satellite $i$.

When the Anti-spoofing is active (as it is the case since January 31 1994), the code observations have a precision ranging from a few decimetres to more than one metre. These measurements are not ambiguous but contain biases called receiver and satellite differential group delays. The existence of these biases is due to the fact that the two GPS frequencies undergo different propagation delays inside the receiver and satellite hardware.

2) the geometry-free combination of dual frequency phase measurements $\Phi_{p, G F}^{i}$;

$$
\Phi_{p, G F}^{i}=\Phi_{p, L 1}^{i}-\frac{f_{L 1}}{f_{L 2}} \Phi_{p, L 2}^{i}
$$

or rewritten in function of the TEC:

$$
\Phi_{p, G F}^{i}=-0.552 \mathrm{TEC}_{p}^{i}+N_{p, G F}^{i}
$$

with $f_{L 1}, f_{L 2}$ the frequency of the $L 1, L 2$ carriers (in $\mathrm{Hz}$ ); $\Phi_{p, L 1}^{i}, \Phi_{p, L 2}^{i}$ the $L 1, L 2$ carrier phase measurements (in cycles) made by receiver $p$ on satellite $i$; $N_{P, G F}^{i}$ a real ambiguity (cycles). 
Phase measurements usually have a precision better than one millimetre but contain an initial ambiguity which is real in the case of the geometry-free combination. In the absence of cycle slips, $N_{P, G F}^{i}$ has to be solved for every satellite pass.

3) a combination of geometry-free code and phase measurements.

$$
P_{p, G F}^{i}-\lambda_{L 1} \Phi_{p, G F}^{i}=\left(D G_{p}-D G^{i}\right)-\lambda_{L 1} N_{p, G F}^{i}
$$

with $\lambda_{L 1}$ the $L 1$ carrier wavelength (in metres).

This third combination is used to solve the ambiguity $N_{P, G F}^{i}$ which is injected in Eq. (4) in order to determine the TEC which is mapped to the vertical. This method which is applied at the Royal Observatory of Belgium allows to combine the advantages of both measurement types: the TEC is obtained from the precise phase measurements but the information contained in the code observations is used to solve the ambiguity. Nevertheless, the procedure requires the determination of the receiver and satellite differential group delays. In most of the cases, these biases have to be computed:

- the satellite biases: they are measured by the manufacturer before the satellites are launched but in most of the cases, these values are not valid any more when the satellites are on their orbit (see, for example, Wilson and Mannucci, 1993; Wanninger et al., 1994; Warnant, 1996).

- the receiver biases: in the past, the old Rogue receivers (the so-called Big-Rogue and Mini-Rogue) had an auto-calibration function allowing to measure the receiver bias. Unfortunately, this function does not exist any more on the Turbo Rogue receiver. To our knowledge, no other receiver has this capability.

In practice, the combined biases (receiver + satellite) can be determined using Eq. (2) where the ionosphere is modelled by means of a polynomial in latitude and local time. In fact, the error made in the determination of the differential group delays is the largest error source when computing the TEC using GPS measurements: these biases can be computed with a precision of about 2-3 TECU. It is clear that they cannot be neglected: for example the bias of one of our Turbo Rogue receivers is +5.33 ns. The fact to neglect it would give an error of 16 TECU on the computed TEC. A detailed discussion of the influence of the biases is given in Warnant (1996) and Warnant (1997).

When the biases have been determined, the TEC which is mapped to the vertical is computed in function of latitude and local time (or longitude) of the ionospheric point. For example, the data collected at Brussels (latitude $=50.8^{\circ} \mathrm{N}$, longitude $=4.4^{\circ} \mathrm{E}$ ) allow to compute the TEC from about $35^{\circ} \mathrm{N}$ to $60^{\circ} \mathrm{N}$ in latitude and from $-20^{\circ} \mathrm{W}$ to $25^{\circ} \mathrm{E}$ in longitude.

To obtain TEC profiles representative of the ionosphere above the observing station, we apply the following procedure:

- we select all the TEC values corresponding to an ionospheric latitude, $L_{\text {iono }}$, given by:

$$
L_{\text {sta }}-1.5^{\circ} \leq L_{\text {iono }} \leq L_{\text {sta }}+1.5^{\circ}
$$

where $L_{s t a}$ is the latitude of the observing station;

- we compute the mean of these TEC values on 15 minute periods.
To verify the reliability of our GPS TEC, we compared it with a independent method: at Dourbes (Belgium), an ionosonde which is the property of the Royal Meteorological Institute of Belgium is collocated with one of our 7 permanent GPS station. This ionosonde produces an electron concentration profile up to the maximum of the $F 2$-layer. The ionosonde measurements are used to compute the Total Electron Content above Dourbes:

- in a first step, numerical integration of the measured bottomside electron concentration profile gives the bottomside part;

- in a second step, analytical integration of a Chapman function modelling the topside electron concentration profile gives the topside part; the parameters of the Chapman function are evaluated using the information contained in measured bottomside profile; we assume that the electron concentration is constant in the protonosphere.

This TEC has been compared with the TEC obtained by GPS on a period of 2 years (1995 and 1996). The results of both methods are in very good agreement: in most of the cases, the difference between "GPS" TEC and "ionosonde" TEC remains within 2-3 TECU; the mean and the standard deviation of the difference computed on this period are respectively 0.46 TECU and 1.72 TECU. More details can be found in Warnant and Jodogne (1998).

\section{Detection of Irregularities in the Total Electron Content}

From Eq. (4), it can be seen that the geometry-free combination also allows to monitor the time variation of the TEC, $\Delta \operatorname{TEC}_{p}^{i}\left(t_{k}\right)$ :

$$
\Delta \operatorname{TEC}_{p}^{i}\left(t_{k}\right)=1.812 \frac{\left(\Phi_{p, G F}^{i}\left(t_{k}\right)-\Phi_{p, G F}^{i}\left(t_{k-1}\right)\right)}{\left(t_{k}-t_{k-1}\right)}
$$

where $\Delta \mathrm{TEC}_{p}^{i}\left(t_{k}\right)$, measured in TECU/min, is defined as:

$$
\Delta \operatorname{TEC}_{p}^{i}\left(t_{k}\right)=\frac{\operatorname{TEC}_{p}^{i}\left(t_{k}\right)-\operatorname{TEC}_{p}^{i}\left(t_{k-1}\right)}{\left(t_{k}-t_{k-1}\right)} .
$$

It is important to stress that the computation of $\Delta \operatorname{TEC}_{p}^{i}\left(t_{k}\right)$ does not require the estimation of the real ambiguity, $N_{p, G F}^{i}$, as long as no cycle slip occurs.

Equation (7) can be used to detect high frequency changes in the TEC due to irregular ionospheric phenomena such as Travelling Ionospheric Disturbances and scintillation effects.

Travelling Ionospheric Disturbances (or TIDs) appear as waves in the electron density (and consequently in the TEC) due to interactions between the neutral atmosphere and the ionosphere. They have a wavelength ranging from a few tens of kilometres to more than thousand kilometres. Their occurrence often cause large gradients in the TEC even on short distances. Scintillation effects are variations in phase and amplitude of a radio signal passing through small-scale irregularities in the ionosphere. Scintillation effects are very often observed in the polar and equatorial regions and are sometimes detected in the mid-latitude regions.

In this paper, we present a method allowing to detect medium-scale Travelling Ionospheric Disturbances (MSTIDs) and scintillation effects using GPS measurements. 
MSTIDs have horizontal wavelengths of several hundreds of kilometres, periods ranging from about 12 minutes to about 1 hour and horizontal phase speeds ranging from 100 to 300 $\mathrm{m} / \mathrm{s}$ (Van Velthoven, 1990).

Travelling Ionospheric Disturbances and scintillation effects cause high frequency changes in the TEC. Consequently, these phenomena can be studied by detecting such changes in $\triangle \mathrm{TEC}_{p}^{i}$.

In order to do that, we filter out the low frequency changes in the TEC by modelling $\triangle \mathrm{TEC}_{p}^{i}$ using a low order polynomial. The residuals $R_{I}$ of this adjustment (i.e. $\Delta \mathrm{TEC}_{p^{-}}^{i}$ polynomial) contain the high frequency terms. Then the standard deviation of the residuals, $\sigma_{R_{I}}$, is computed for every observed satellite, using periods of 15 minutes of measurements. When $\sigma_{R_{I}}>0.8$, we decide that an "event" is detected. Such "events" are presented in Fig. 1. This figure shows the gradients, $\triangle \mathrm{TEC}$, observed in 3 GPS stations operated by the Royal Observatory of Belgium. These gradients are due to a (Medium-Scale) Travelling Ionospheric Disturbance. This TID is detected as 4 "events": indeed, as a consequence of the TID occurrence, $\sigma_{R_{I}}$ remains above the threshold value of $0.08 \mathrm{TECU} / \mathrm{min}$ during 4 periods of 15 minutes. In fact, most of the "events" detected in Belgium are due to TIDs but scintillation effects are sometimes observed, in particular after severe geomagnetic storms. The detection software can easily decide if the high frequency changes in the TEC are due to scintillations or TIDs because the signature (period) of these 2 phenomena are very different.

With a sampling interval of 30 seconds, 24 hours of GPS measurements in the RINEX format are stored in a file of which the size is ranging from $1.5 \mathrm{Mb}$ to more than $2 \mathrm{Mb}$. To perform any long term study based on GPS measurements,
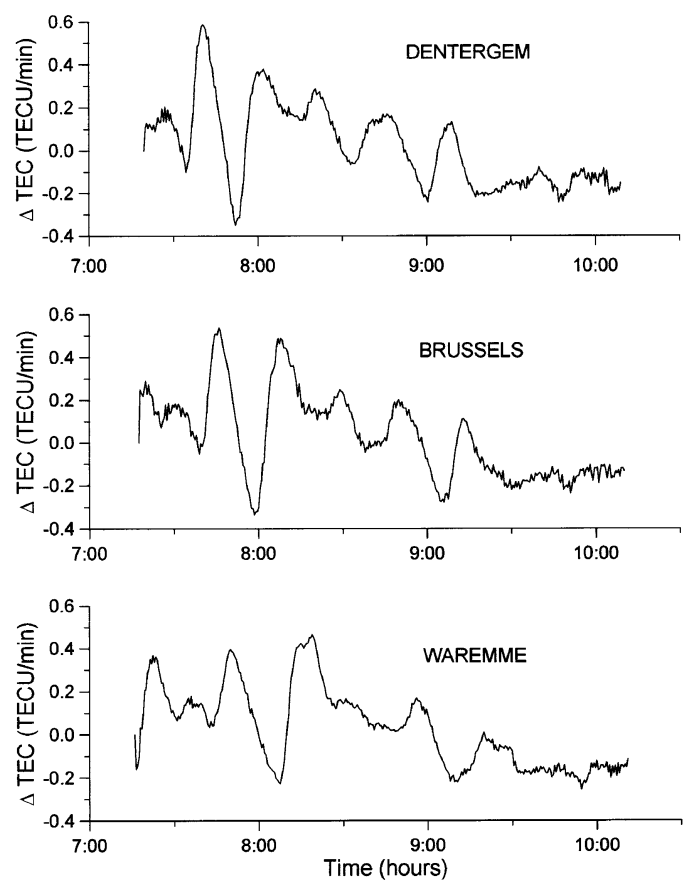

Fig. 1. TEC gradients due to a TID observed on October 301994 in 3 Belgian stations. it is thus indispensable to develop automatic data processing procedures. In particular, it is not realistic to imagine that an operator could screen the residuals to decide "manually" if a TID is present or not. For this reason, we must choose threshold values which will be used by the computer to take an automatic decision. The choice of $0.08 \mathrm{TECU} / \mathrm{min}$ as threshold value to decide if a an event is taken into account comes from the fact that the multipath can also give rise to high frequency changes in the geometry-free combination. This site-dependent effect can reach several centimetres on phase measurements and has periods ranging from a few minutes to several hours depending on the distance separating the reflecting surface from the observing antenna (if this distance is shorter, the period is longer). The multipath effect being more frequent at low elevation, we have chosen an elevation mask of $20^{\circ}$. In the case of the Belgian permanent GPS network, a threshold value of $0.08 \mathrm{TECU} / \mathrm{min}$ is large enough to avoid to interpret multipath effects as ionospheric phenomena. This value should be valid for most of the GPS sites but should be applied with care in locations where the multipath is particularly important. An additional verification is then performed: the comparison of the ionospheric variability observed in neighbouring $(50 \mathrm{~km})$ GPS stations allows also to distinguish between multipath and ionospheric phenomena: indeed, large residuals observed at the same time in different stations cannot be due to multipath.

Two other error sources can affect our method: cycle slips
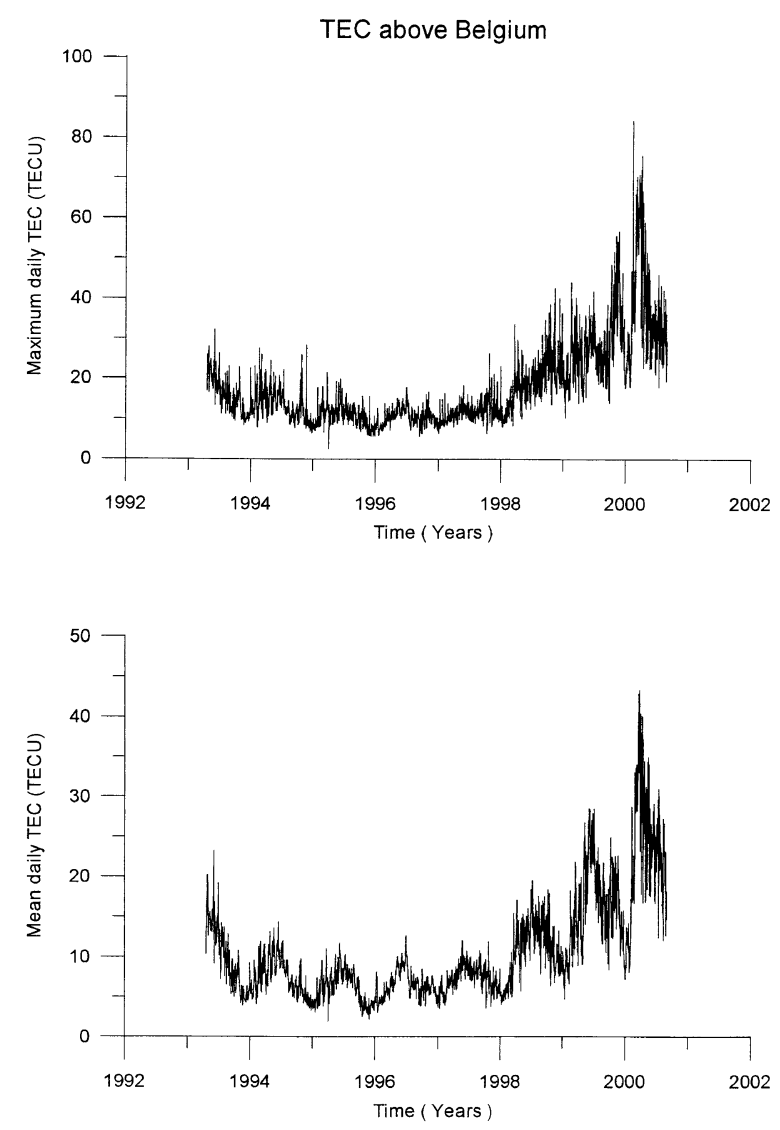

Fig. 2. Daily maximum and daily mean TEC at Brussels from April 1993 to August 2000. 

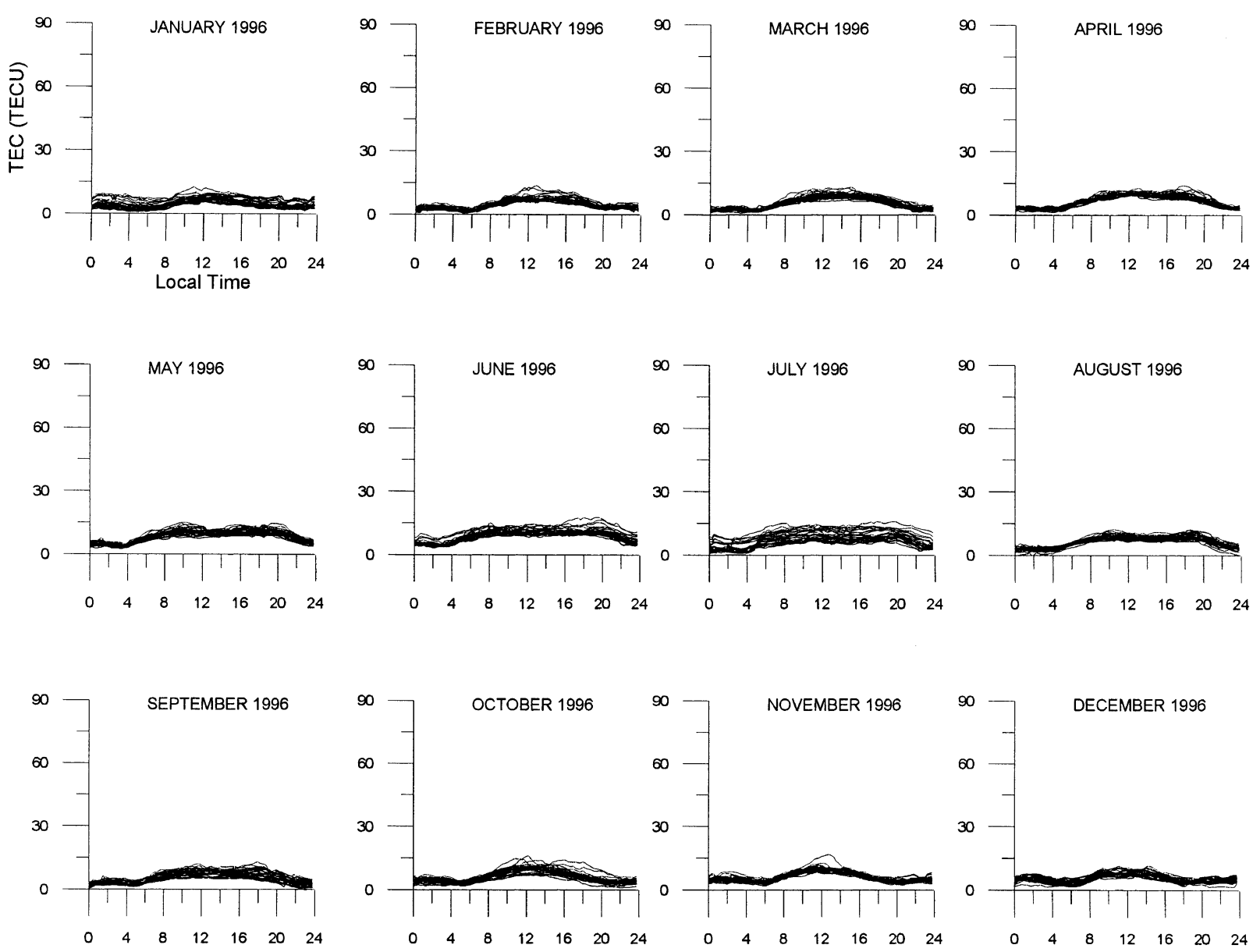

Fig. 3. TEC at Brussels in 1996.

and phase surges. Cycle slips are jumps of an integer number of cycles which occur when the receiver loses lock on the satellite signal. In an automated data processing procedure, an uncorrected cycle slip could result in a $\sigma_{R_{I}}$ above the threshold value even if no ionospheric perturbation is present. Again, interstation comparison can help to solve the problem.

Phase surges are experienced by receivers using the crosscorrelation technique to process the GPS signal when the Anti-spoofing is active. These phase surges give rise to several successive jumps in the $L 2$ phase measurements. These jumps are not integer numbers of cycles. For this reason, this error is much more difficult to detect. In addition, this effect is related to the receiver-to-satellite geometry; it means that a similar effect can be observed in several neighbouring GPS stations at the same time: the problem occurs when 2 satellites have the same Doppler as seen by the receiver (Sleewaegen, 1999). Nevertheless, these jumps can be identified because their signature and in particular their period is always shorter (a few minutes) than the period of the MSTIDs.

The choice of 15 minute periods to compute the ionospheric variability is due to the fact that most of the MSTIDs have periods ranging from 5 to 30 minutes. If we choose a too short period, the TID will not have the time to cause TEC changes large enough to be detected. On the other hand, if the period is too long, the large residuals in $\triangle \mathrm{TEC}_{p}^{i}$ due to the
TID will be lost among the other residuals and the resulting $\sigma_{R_{I}}$ will remain under the threshold of $0.08 \mathrm{TECU} / \mathrm{min}$.

\section{Results}

\subsection{TEC behaviour}

The solar activity has reached its 11-year activity cycle minimum in May 1996. Since the end of 1997, the solar activity is increasing very quickly giving rise to a stronger ionospheric activity. The solar maximum will probably be reached during the year 2000 or 2001 . The TEC dependance on the solar cycle can be easily seen from Fig. 2 which displays the daily maximum and daily mean TEC from April 1993 to August 2000.

Figures 3 and 4 show respectively the TEC observed at Brussels from January 1996 to December 1996 (solar minimum) and from September 1999 to August 2000 (at or close to solar maximum). On these 2 figures, all the daily TEC profiles relative to one month are represented on the same graph.

From these figures, we can see that the TEC seasonal behaviour depends on the solar cycle:

- the largest daily mean TEC values are observed during the summer except in 2000 where these largest values are measured during the spring.

- at solar minimum, the daily maximum TEC ranges from about 5 TECU to 15 TECU; at solar maximum, it ranges 

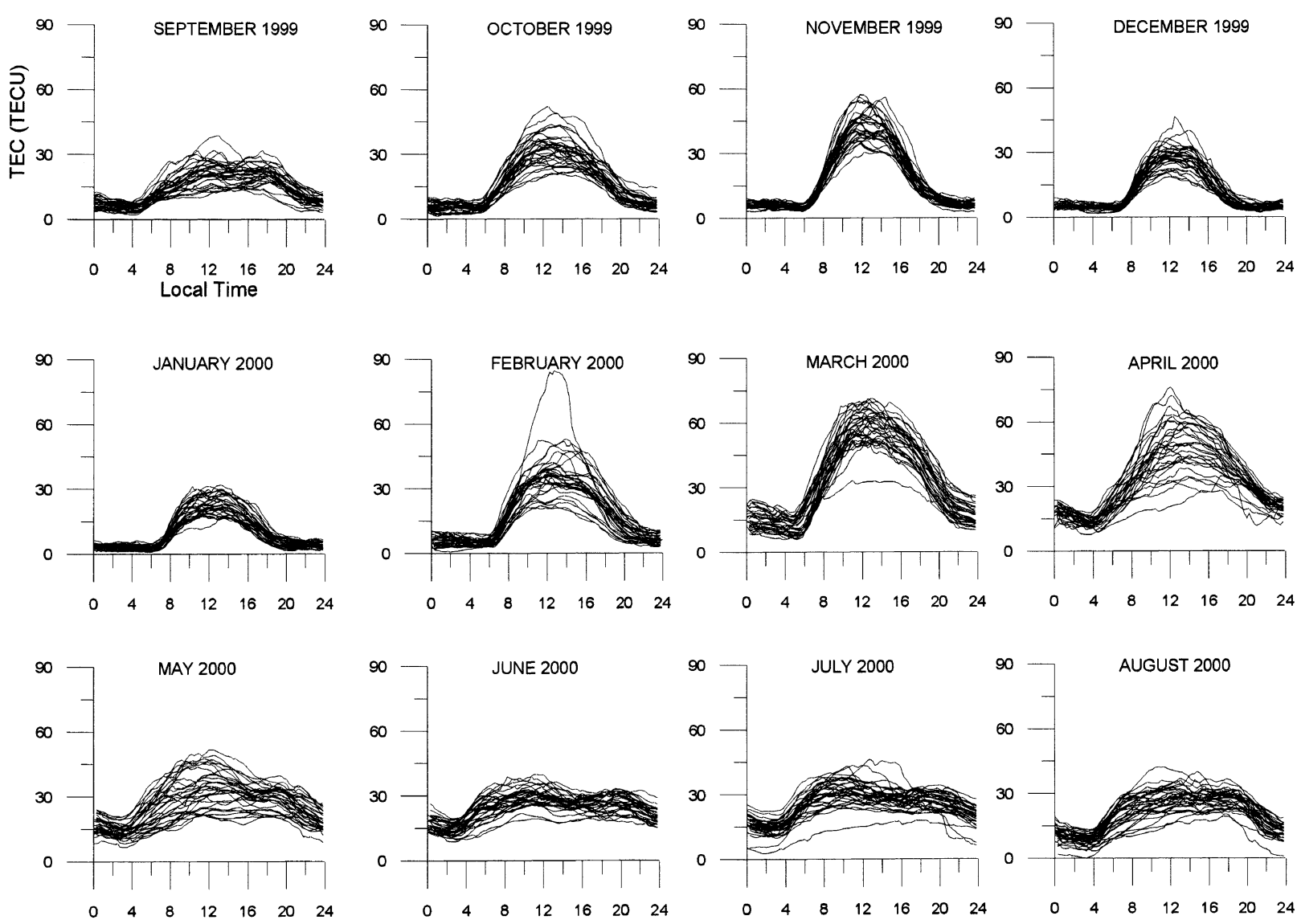

Fig. 4. TEC at Brussels from September 1999 to August 2000.

between about 20 to 85 TECU;

- at solar maximum, the daily maximum TEC in November is about 4 times larger than at solar minimum, in March, it is about 6 times larger but during the summer (June-July) it is 2-3 times larger.

In addition to that,

- at solar maximum, the highest TEC value observed at Brussels was close to 85 TECU; at solar minimum, the TEC is most of the time smaller than $10 \mathrm{TECU}$ and is often of the order of 1 TECU at night.

- independently of the solar cycle, the largest daily maximum TEC values are observed in February-March and in October-November.

Most of our results are in good agreement with the results obtained by Soicher (1988). Nevertheless, at solar maximum, Soicher observed the occurrence of a very pronounced secondary maximum in the TEC at about 18:00 UT. We did not observe this maximum which is probably due to the fact that Soicher based his study on measurements made at Haifa in Israel which is much closer to the magnetic equator than Brussels.

\subsection{Statistics concerning the occurrence of TIDs}

Figure 5 shows the number of events due to TIDs observed per month at Brussels from April 1993 to August 2000. On this figure, we can see that:

- TIDs are frequently observed all the time (during all seasons and during all phases of the solar cycle).

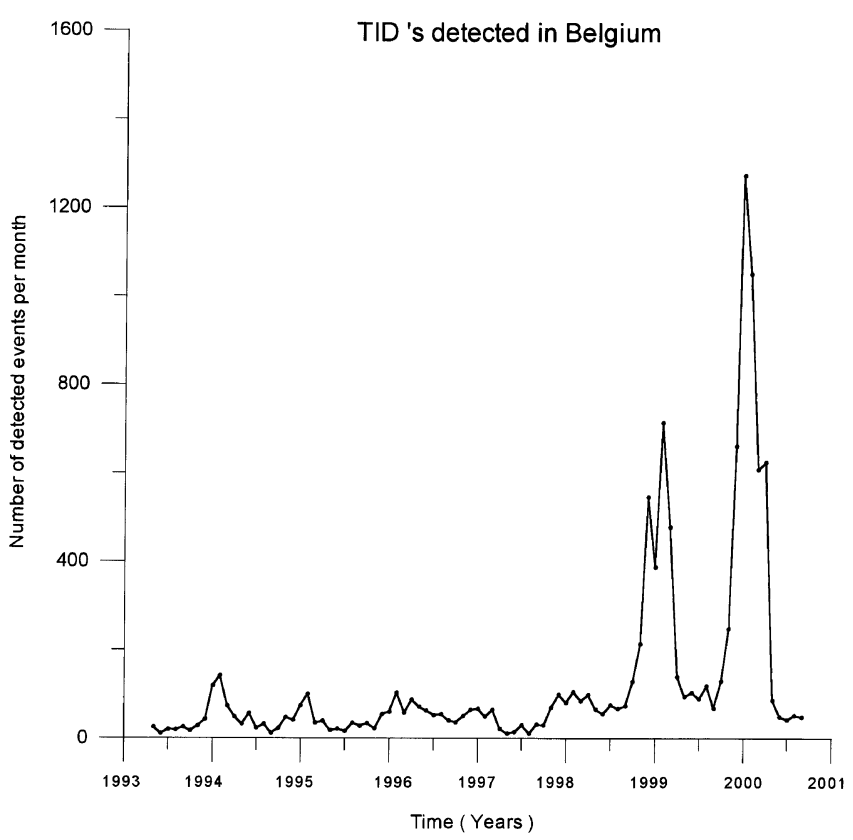

Fig. 5. Number of "events" detected per month at Brussels from April 1993 to August 2000. 
- there is an annual peak in the number of TIDs during the winter independently of solar activity but this peak is much sharper at solar maximum.

- the number of TIDs strongly depends on solar activity: in January 1996, 200 events were detected; in January 2000, 1300 events were observed.

These results confirm the conclusions of previous studies performed by means of other independent techniques (see for example, Van Velthoven, 1990).

\section{Conclusions and Further Work}

The paper has outlined a method allowing to compute the Total Electron Content and to detect ionospheric irregular phenomena such as TIDs and scintillations. The goal of the work is to build a data set which can serve as reference to identify possible correlations between the ionospheric activity and unidentified problems in GPS time series or GPS data processing. The paper has described the effect of the increasing solar activity on the ionosphere: since 1998, larger TEC values are observed; in February 2000, the TEC has reached the level of 85 TECU; at solar minimum, the TEC remains smaller than 10 TECU in most of the cases. In addition, the number of detected TID's has also tremendously increased to reach the level of 1300 events per month in January 2000; the number of events was about 200 in January 1996. The results which are in good agreement with previous independent studies show that TIDs are very common phenomena at Brussels.

As further steps we will:

- make use of 1 second data in order to detect smaller-scale ionospheric irregular phenomena.

- have a closer look to the correlation between ionospheric irregular phenomena and geomagnetic activity.

- based on our reference ionospheric data set, we will study the influence of the ionospheric activity on GPS position time series.

\section{References}

da Rosa, A. V., H. Waldman, J. Bendito, and O. K. Garriott, Response of the ionospheric electron content to fluctuations in solar activity, J. Atmos. Terr. Phys., 35, 1429-1442, 1973.

Dehant, V. and P. Pâquet, Modeling of the apparent height variations of a tranet station, Bulletin Géodésique, 57, 354-364, 1983.

Feitcher, E. and R. Leitinger, A 22-year cycle in the F layer ionization of the ionosphere, Ann. Gephysicae, 15, 1015-1027, 1997.

Lanyi, G. E. and T. Roth, A comparison of mapped and measured total ionospheric electron content using global positioning system and beacon satellite observations, Radio Sci., 23, 483-492, 1988.

Sleewaegen, J.-M., Surge Anomaly in Cross-Correlated GPS measurements: Description and Analysis, Navigation, 46(2), 119-125, 1999.

Soicher, H., Traveling ionospheric disturbances (TIDs) at mid-latitudes: solar cycle phase dependence, Radio Sci., 23, 283-291, 1988.

Van Velthoven, P. J., Medium-scale irregularities in the ionospheric electron content, Ph.D. Thesis, Technische Universiteit Eindhoven, 1990.

Wanninger, L., Der Einfluß der Ionosphäre auf die Positionierung mit GPS, Ph.D. Thesis, Wissenschaftliche Arbeiten der Fachrichtung Vermessungswesen der Universität Hannover, Nr. 201, 137 pp., 1994.

Wanninger, L., E. Sardón, and R. Warnant, Determination of the Total Ionospheric Electron Content with GPS-Difficulties and their Solution, Proceedings of Beacon Satellite Symposium '94, edited by Dpt. of Physics of University of Aberystwyth, 13-16, 1994.

Warnant, R., Etude du comportement du Contenu Electronique Total et de ses irrégularités dans une station de latitude moyenne. Application aux calculs de positions relatives par le GPS, Ph.D. Thesis (in French), Série Géophysique ( $\mathrm{N}^{\circ}$ Hors-Série) de l'Observatoire Royal de Belgique, Bruxelles, 1996.

Warnant, R., Reliability of the TEC computed using GPS measurements: the problem of hardware biases, Acta Geodaetica et Geophysica Hungarica, 32(3-4), 451-459, 1997.

Warnant, R. and J.-C. Jodogne, A Comparison between the TEC Computed using GPS and Ionosonde Measurements, Acta Geodaetica et Geophysica Hungarica, 33(1), 147-153, 1998.

Wilson, B. D. and A. J. Mannucci, Instrumental Biases in Ionospheric Measurements derived from GPS data, Proceedings of ION GPS' 93, Salt Lake City, 1993.

R. Warnant (e-mail: R.Warnant@oma.be) and E. Pottiaux 